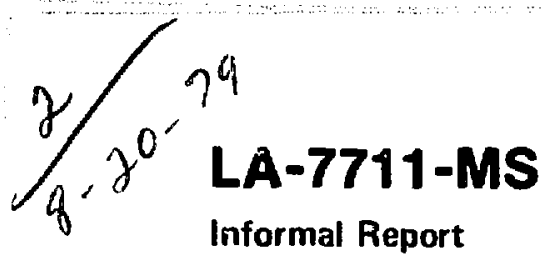

NEPS Reactor and Heat Exchanger

Summary Status (December 1978) 


\title{
NEPS Reactor and Heat Exchanger Summary Status (December 1978)
}

\author{
Daniel R. Koenig
}
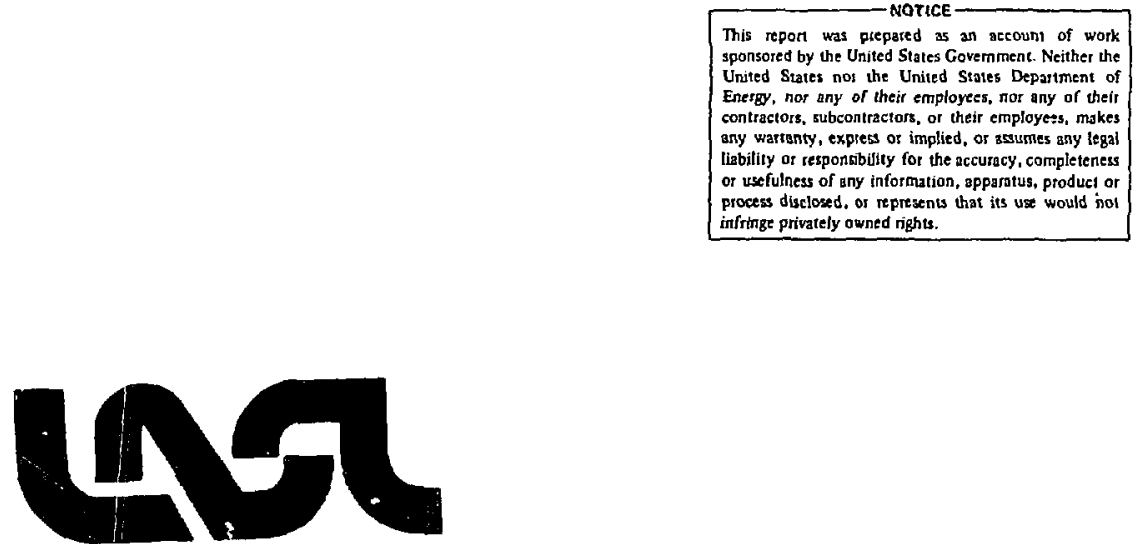


\title{
NEPS REACTOR AND HEAT EXCHANGER \\ SUMMARY STATUS (DECEMBER 1978)
}

\author{
by
}

Daniel R. Koenig

\begin{abstract}
The design of a $3 \mathrm{MW}_{\mathrm{t}}$ neat-pipe cooled nuclear reactor for electric propulsion of spacecrafts has been evolving continually. Several modifications nave been made to make the reactor design more tolerant of potential heat pipe failures. Design parameters and operating cnaracteristics for the moditied reactor are presented.

The desirability of separating the reactor assembly from the thermionic converter assembly has been recognized, and an intermediate heat exchanger to couple the two subassemblies together has been conceptualized.
\end{abstract}

\section{INTRODUCTION}

The reference core design for the 3-iWW Nuclear Electric Propulsion System (NEPS) reactor contains 90 molybdenum/lithium heat pipes. These heat pipes penetrate the radiation shield before transferring their heat to thermionic converters triat are bonded onto the condenser end of the heat pipes.

Detailed consideration of the consequences of core heat pipe failure has indicated the desirability of increasing tire number of heat pipes in the core. It has also focused attention on the need to modify the periphery of the core to avoid excessive fuel temperatures in case of neat pipe failure there. Increasing the number of core heat pipes has rekindled the desirability of decoupling the core from the thermionic converters by having separate sets of heat pipes, optimizea independently, for each of these two subassemblies. Accordingly, it has motivated the design of a heat exchanger to couple these two sets of heat pipes. 
This report reviews the current status of the NEPS reactor design and describes the conceptual design of a heat exchanger between the core and the thermionic converters.

\section{EFFECT OF HEAT PIPE FAILURE ON FUEL TEMPERATURE}

The fuel temperature rise in the vicinity of a failed core neat pipe was determined from two-dimensional finite element heat transfer calculations as exemplified in Fig. 1. Two kinds of heat pipe failures were analyzed, failure of an internal heat pipe which is surrounded by six functioning neighbors, and failure of a corner, periphery heat pipe, modeled in Fig. 1A, which has only three neighbors. Unless special provisions are taken on the core periphery, the latter failure produces unacceptably high temperatures near the failed heat pipe as shown in Fig. 1B. This is because heat generated on the outer edge of the failed periphery element has to travel a long and narrow path around the failed pipe before reaching a functioning heat pipe. Design modifications of the core periphery to avoid this situation are discussed below.

The maximum fuel temperature resulting from failure of an internal heat pipe is plotted in Fig. 2 vs the number of core heat pipes. Also shown in Fig. 2 are the average and maximum fuel temperatures in a normally functioning fuel element. The fuel temperdture near t,e failed heat pipe shown in this figure assumes perfect thermal contact between adjacent fuel elements and it must de increased by the temperature arop expected across the inter$f$ ace of adjacent fuel elements. This temperature drop is difficuit to calculate out reasonable estimates are in the range of 50-300 $\mathrm{K}$ for a 90 element core, placing the maximum temperature uncomfortatly close to the melting point of molybuenum $(2900 \mathrm{~K})$. Raising the number of core i:eat pipes to $16 \%$, decreases the fuel temperature very significantly without unduly increasing the complexity of the core. The number 162 corresponds to a perfect hexagonal assembly with the central and the six corner elements removed. Removing the corner elements reduces the maximum core diameter considerably and greatly eases the circularization of the core assembly. The center element is removed for symmetry reasons. Design parameters and operating characteristics for a 162 heat pipe core are described in the parameters section. 


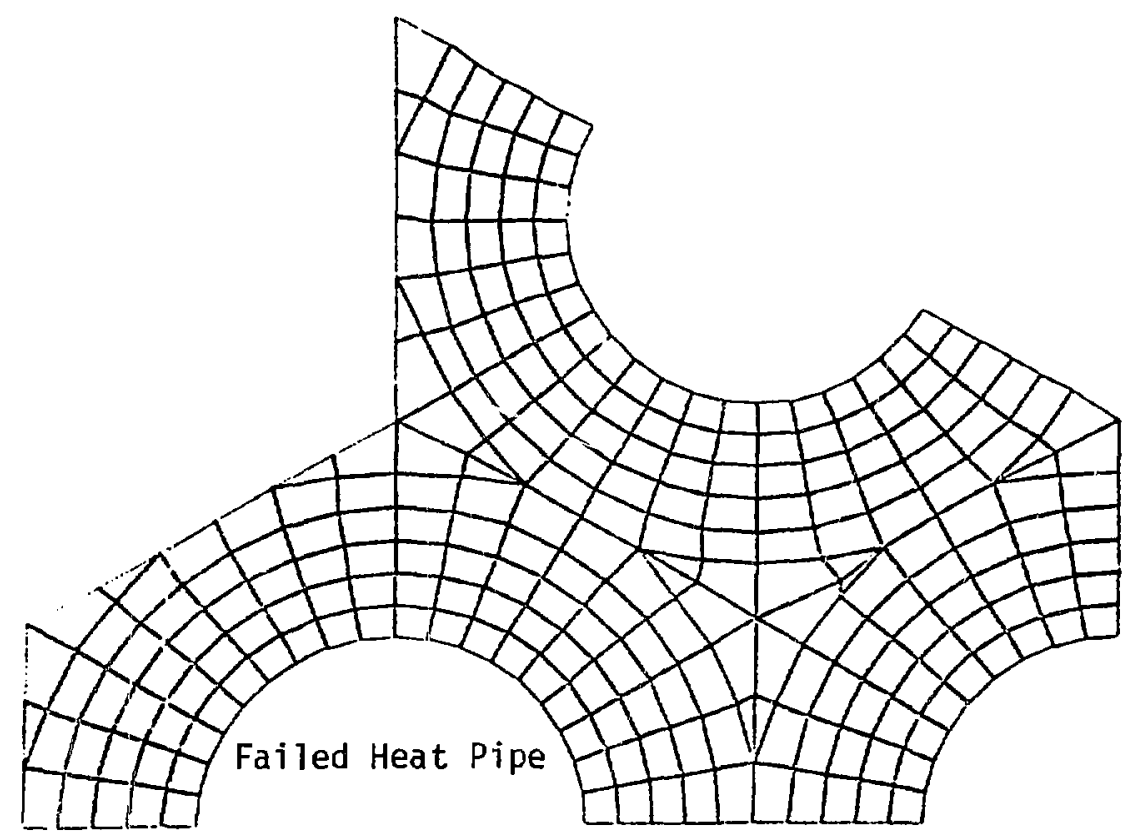

A. Calculational array for periphery heat pipe failure.

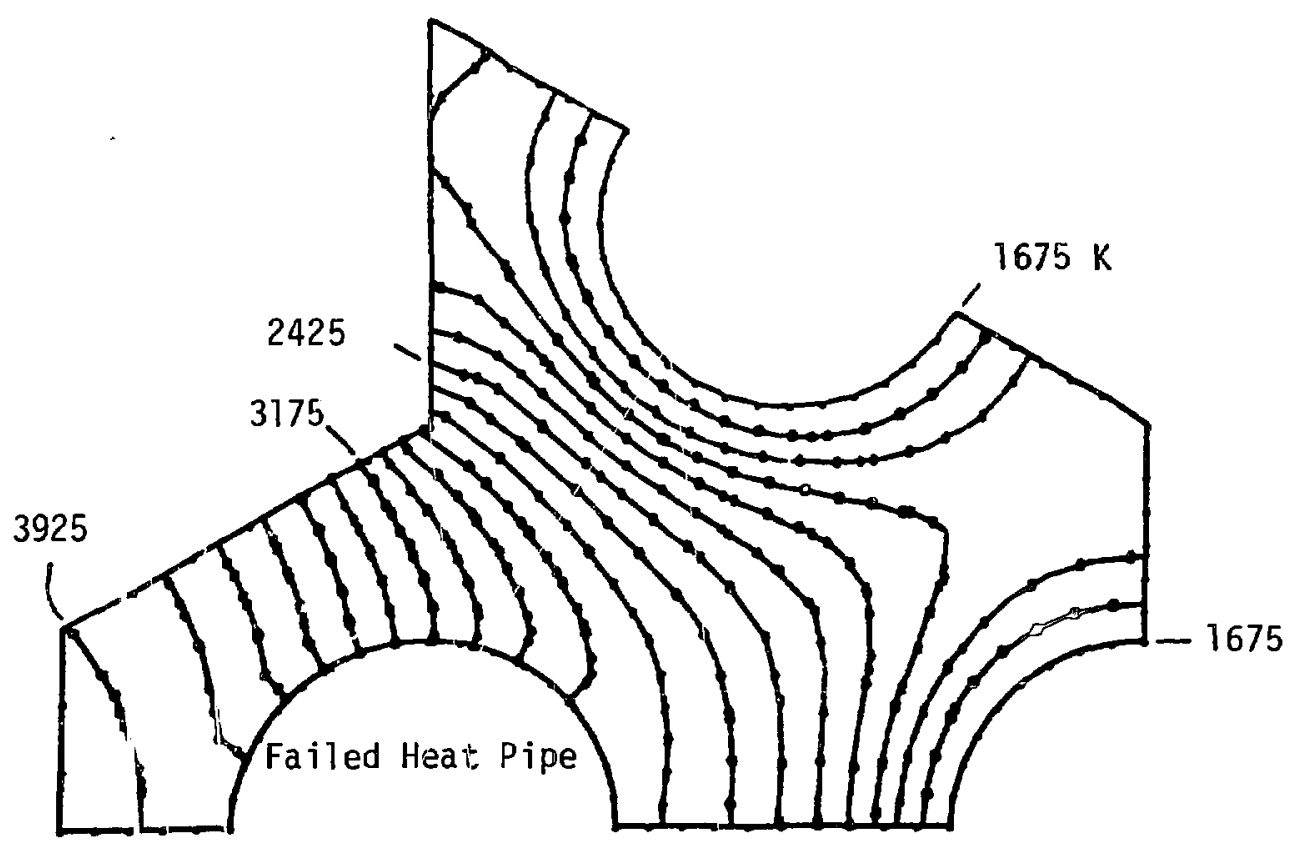

B. Calculated isotherms for periphery failure.

Fig. 1. Heat transfer of a heat-pipe failure at a corner of the core periphery. The straight bounciaries are assumed to be adiabatic. 


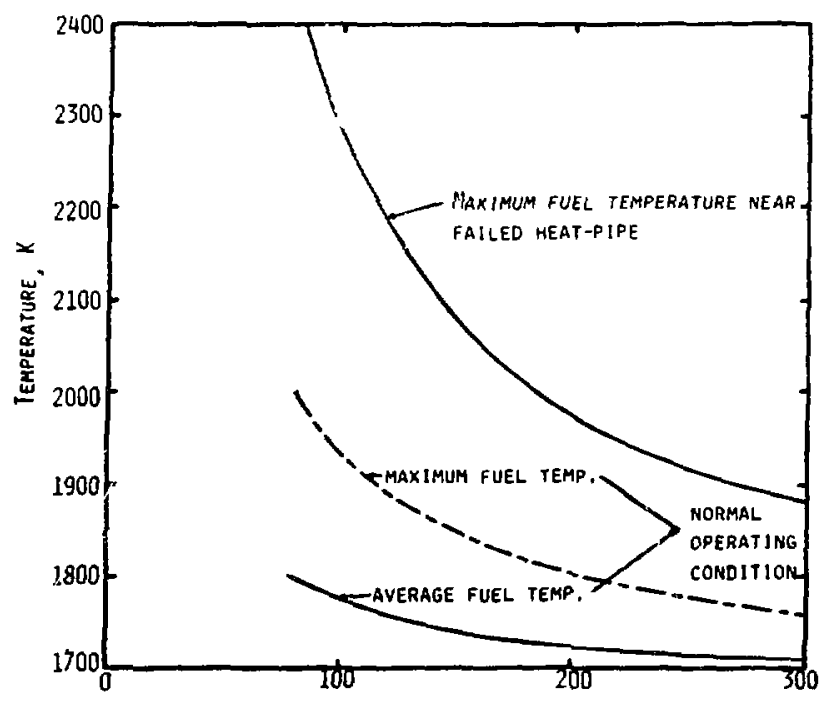

Fig. 2. Fuel temperatures vs the number of heat pipes in the core.

\section{FUEL ELEMENT DESIGN}

The reference fuel element is still the $\mathrm{UO}_{2}$ pellet in a molybdenum matrix design, shovn schematically in Fig. 3. The neat pipe bonded at the center of the element has a wick structure consisting of axial grooves formed in the pipe wall and covered with a fire molybcienum screen. The heat pipe fluid is lithium. This kind of wick structure provides many low impedance, redundant paths for returning the liquid lithium from the cunuenser to the evaporator. The screen wick is easy to fabricate and if a good bond can be obtained between the screen and the land areas between the grooves,

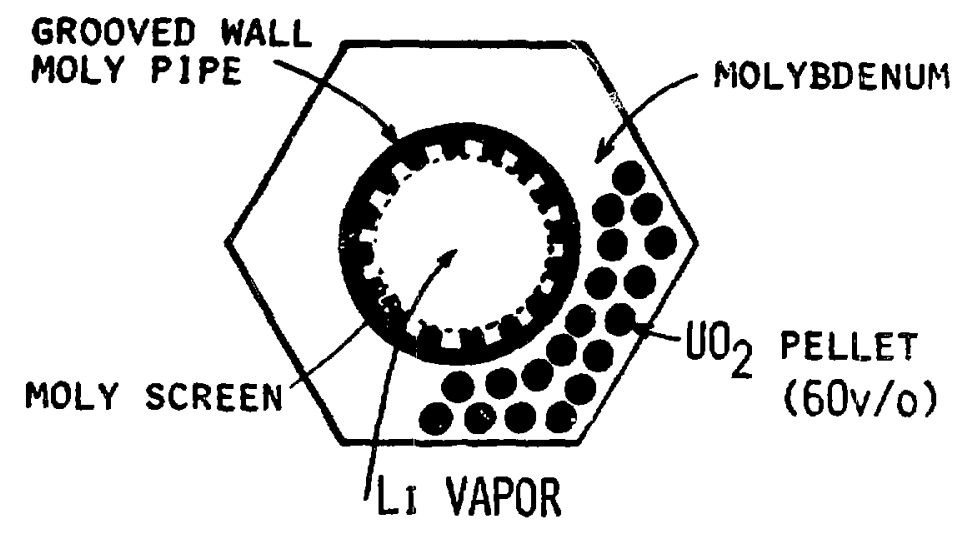

Fig. 3. Schematic drawing of " $\mathrm{UO}_{2}$ pellet" fuel element design. 
it appears that this heat pipe can be bent relatively easily as required in the overall power plant cesign. There are several drawbacks to this neat pipe design, however. Because the wall is thick in order to accommodate the grouves, the pipe is relatively heavy and the radial temperature arop across the pipe wall is several times larger than it could be. Also an adequate fabrication technique for forming the grooves has rot yet been ceveioped. Consequently, a back-up heat pipe design has been selected which consists of a smooth tube lined with a distributive screen wick to that several (two to six) screen arteries are attached. This heat pipe design has a much smaller wall thickuess but fewer redundant paths for liquid flow. All the components are easy to fabricate but bonding the arteries to the screened wall is difficult and a technique for bending the wick assembly nas not been developed.

The molybdenum matrix, $\mathrm{UO}_{2}$ pellet design can accommodate the range of $\mathrm{UO}_{2}$ concentration (47-70 vol\%) required to achieve a flat (to $\pm 5 \%$ ) radial power profile in the core. However, as discussed below, une way of alleviating the generation of excessite fuel temperatures on the core periphery involves achieving a radial power density profile that is peaked on the periphery. Obtaining this power density profile may require raising the $\mathrm{UO}_{2}$ concentration on the periphery to 80 vol\% or more. Such a high $\mathrm{UO}_{2}$ conceritration cannot be obtained in the pellet design without loweririg excessiveiy the effective thermal conductivity of the fuel element. Therefore,

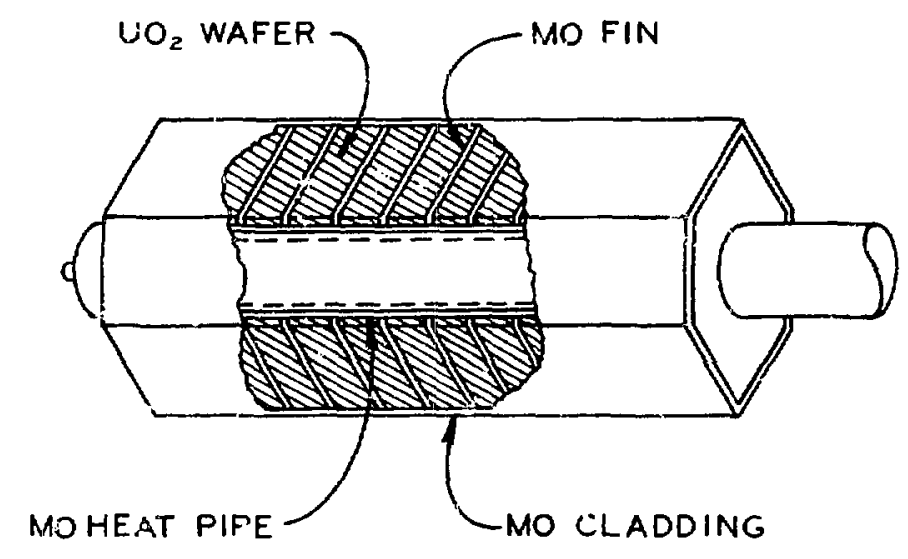

Fig. 4. Schematic drawing of " $\mathrm{UO}_{2}$ wafer" fuel element design. 
another fuel element design is being investigated that inherently has a better thermal conductivity than the pellet design and that may also be easier to fabricate. The design concept, referred to as the " $\mathrm{UO}_{2}$ wafer" fuel element, is illustrated in Fig. 4. The wafer fuel element consists of hexagonal $\mathrm{UO}_{2}$ wafers which are slipped over the heat pipe and sandwiched between molybdenum fins. The entire fuel region is clad in molybdenum. The fin arrangement places all (except for the cladding) the molybdenum in a direction that serves to conduct the heat generated in the $\mathrm{UO}_{2}$ toward the heat pipe and tnereby achieves a better effective radial thermal conductivity. A good bond between the $\mathrm{UO}_{2}$ and the heat pipe is not essential beciuse nearly ail the heat is transferred to the pipe by the fins. In any case the $\mathrm{UO}_{2}$ will redistribute itself eventually, by evaporation and condensation, against the heat pipe which is the coldest part of the fuel element. A good thermal bond between the fins and the heat pipe is desirable and should not be difficult to obtain.

\section{CORE DESIGN}

The core consists of a hexagonal assembly of 162 heat-pipe fuel elements as shown in a partial cross-sectional view in Fig. 5. Trie centra? and

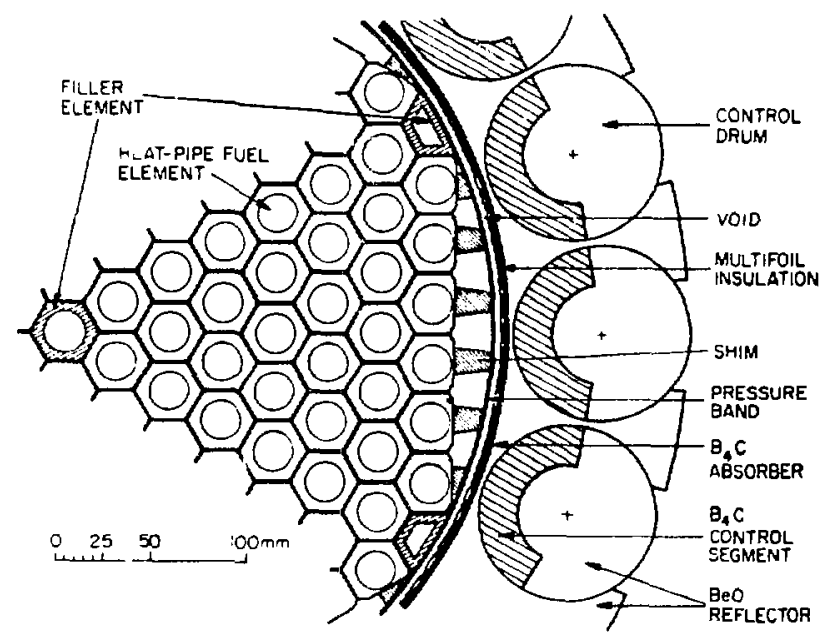

Fig. 5. Cross section of reactor. Periphery fuel elements have no fuel on trieir outer edge. 
corner elements are filler elements consisting only of short, core-length heat pipes. The periphery elements have been sliced off flush with the heat pipe so that they contain no fuel along the ir outer edge.

The entire core is held tightly with several circumferential pressure bands. This assembly is surrounded on all sides, first by multifoil thermal insulation, followed by a thin layer of thermal neutron absorber $\left(B_{4} C\right)$, and a $B e 0$ reflector. The purpose of the absorber is to adjust power peaking on the periphery of the core caused by low-energy neutrons reflected from the $\mathrm{Be} 0$. The radial reflector contains rotating drums loaded with $\mathrm{B}_{4} \mathrm{C}$ for control of reactivity.

The configuration of the periphery fuel elements ensures that failure of a periphery heat pipe will not produce higher fuel temperatures thian would result from failure of an internal heat pipe. If the radial power density profile is adjusted to be flat, the peripiery ieat pipes would carry about $30 \%$ less power than the internal ones, corresponding to the amount of fuel that has been removed from the outer edge of the periphery elemerits. This reduced power may complirate the design of the thermionic converter assembly. However, it is estimated that about half of the power reduction can be recovered, with only a modest increase in fuel $\Delta T$, by adjusting the power density profile to actually peak on the core periphery. This adjustment can be accomplished by increasing the fuel concentration on the periphery to $80 \mathrm{vol}^{\mathrm{N}} \mathrm{UO}_{2}$ and by reducing the thickness of the stationary $\mathrm{B}_{4} \mathrm{C}$ layer, whose purpose is to affect the power profile on the periphery. Increasing the $\mathrm{UO}_{2}$ concentration to 80 vol\% cannot practically be done with the pellet fuel element design isut it could be dune with the wafer design described above. Finally, the use of a heat exchanger, such as the one described below, between the core and the converter heat pipes, would enable further smcothing of the power delivered to each converter heat-pipe to within about $\pm 5 \%$ of the average power.

An alternative to the treatment of the core periphery just described is shown in Fig. 6. Here, the periphery fuel elements have been squared off tut they have the same fuel volume as internal elements. In case of a heat pipe failure, heat generated in the outer edge of periphery elements is distributed to nearby periphery elements by circumferential heat pipes located on the flat faces of the core. Thermal contact between the core and the circumferential heat pipes, which have rectangular cross sections, is achieved 


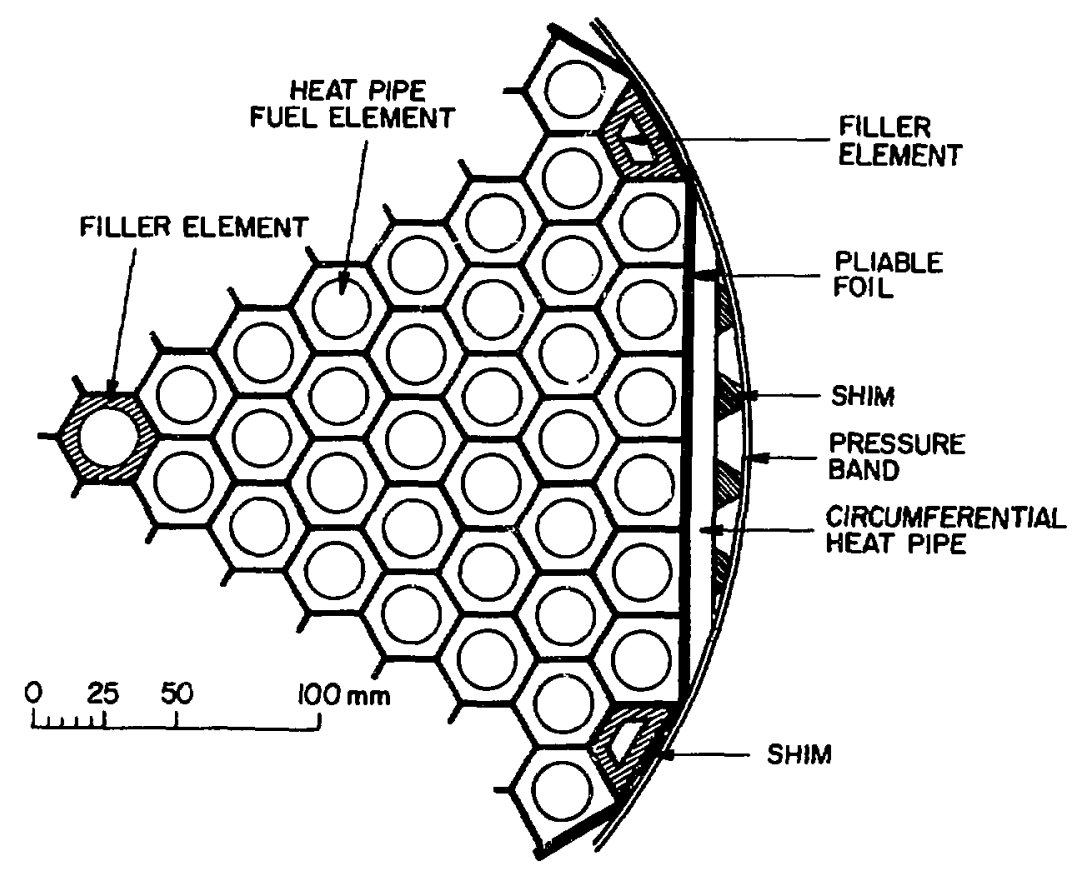

Fig. 6. Cross section of core showing circumferential periphery heat pipes. Periphery fuel elements are fully loaded with fuel.

with deformable foil. The circumferential heat pipes are held against the core by a set of pressure bands. Power flattening is easier to achieve in this alternative concept; however, the core design is greatly complicated by the additional set of heat pipes.

In concluding this section it must be pointed out that, with or without the use of an intermediate heat exchanger, the thermionic converter system design must have the flexibility to accommodate the failure of core heat pipes.

\section{DESIGN PARAMETERS FOR A 162 HEAT PIPE CORE}

Design parameters and operating characteristics have been computed for a core cooled by 162 heat pipes. Fuel element dimensions are listed in Table I, reactor dimensions and weights are shown in Tables II and III, respectively, and the operating characteristics are described in Table IV. 
The reactor dimensions and weights are essentially unchanged from the 90 heat pipe core design except for the heat pipe weight which has been reduced almost in half. Previous estimates of this weight had been overly conservative. The increased number of core heat pipes results in a nearly $50 \%$ reduction of the temperature gradient in the fuel. The lower average fuel temperature has the effect of decreasing the estimated fuel swelling to 5 vol\%.

\section{TABLE I}

DESCRIPTION OF 60 v/o U0 2 - 40 v/o MO HEAT PIPE FUEL ELEMENT FOR A 162 ELEMENT CORE

Width across hexagonal flats (mm) 31.8

Equivalent mean diameter (mm) 33.4

Length of fuel section (mm) 427

Mo heat pipe outer diameter $(\mathrm{mm}) \quad 20.0$

Vapor passage effective diameter $(\mathrm{mm}) \quad 15.5$

Vapor passage area $\left(\mathrm{mm}^{2}\right) \quad 188$

Fuel region vol. fraction (includes porosity) 0.642

${\mathrm{Mo}-U \mathrm{U}_{2}}_{2}$ v01. fraction (assuming $100 \%$ dense) 0.578

Total heat pipe volume fraction 0.358

Heat pipe wail and wick volume fraction 0.143

Heat pipe vapor volume fraction $\quad 0.215$

Fuel porosity and swelling allowance fraction 0.064 
TABLE II

REACTOR DIMENSIONS

Equivalent core diameter (mm) 427

Equiva?ent reactor diameter (mm) 657

$\begin{array}{ll}\text { Core length }(\mathrm{mm}) & 427\end{array}$

Reactor length, (mm) 637

Nominal reflector thickness $(\mathrm{mm}) \quad 800$

$\begin{array}{ll}\text { Pitch spacing (mm) } & 31.8\end{array}$

Number of heat pipe fuel elements 162

Core void fraction (center hole), \% 0.6

Core non-fuel volume fraction $\quad 0.426$

Core $60 \mathrm{v} / 0$ v0 - $40 \mathrm{v} / 0$ Mo vo?, fraction 0.574

(assuming $100 \%$ dense $\mathrm{UO}_{2}$ Mo fuel)

REACTOR WEIGHT SUMMARY, $(\mathrm{kg})$

$\mathrm{UO}_{2}$ - Mo fuel, total weight

372

$\mathrm{UO}_{2}$ on ly

${ }^{235} \mathrm{U}$ on $1 \mathrm{y}$

189

90 Mo heat pipes $(110 \mathrm{~kg} / \mathrm{m})$

(to outer edge of reactor on 1y)

Be0 reflector

Control system $\quad 75$

Reactor support structure (assume 5\%)

$\begin{array}{ll}\text { TOTAL } & 950\end{array}$ 
TABLE IV

REACTOR OPERATING CHARACTERISTICS FOR A 162 ELEMENT CORE

Nominal electrical power output (kWe) 400

Thermal power level (MWth) 3.05

Thermionic converter efficiency (\%) 15

Life time at full power (h) $\quad 75,000$

Number of fuel elements 162

Average power density in $\mathrm{UO}_{2}-$ Mo $\left(\mathrm{MW} / \mathrm{m}^{3}\right.$ or $\left.\mathrm{W} / \mathrm{cm}^{3}\right) \quad 78$

Average power density in $\mathrm{UO}_{2}\left(\mathrm{MW} / \mathrm{m}^{3}\right.$ or $\left.\mathrm{W} / \mathrm{cm}^{3}\right) \quad 130$

Power per heat pipe $(\mathrm{kW}) \quad 18.8$

Heat pipe axial heat flux $\left(\mathrm{MW} / \mathrm{m}^{2}\right) \quad 100$

Heat pipe radial heat flux $\left(M W / m^{2}\right) \quad 0.91$

Heat pipe temperature $(K) \quad 1675$

Average fuel temperature (K) 1738

Maximum fuel $\Delta T\left(K{ }^{*}\right)$

Maximum fuel temperature $\left(K^{\star}\right) \quad 1835$

${ }^{235} U$ burn-up (\%) $\quad 5.8$

Fission density in $\mathrm{UO}_{2}-\mathrm{Mo}_{0}\left(10^{20}\right.$ fissions $\left./ \mathrm{cm}^{3}\right)$

Fuel volume swelling (\%) 5

Reactivity change $\Delta k$ Due to fuel burn-up (\%) 3.5

Reactivity change $\Delta k$ due to thermal expansion (\%) $\quad 1.5$

*Assumes a 1.5 peak-to-average power density ratio

\section{HEAT EXCHANGER DESIGN}

An alternative to mounting the thermionic converters directly onto the condenser section of the core heat pipes is to assemble the converters on a separate set of heat pipes that are coupled to the core heat pipes with an intermediate heat exchanger. Several concepts to accomplish this thermal coupling are illustrated in Fig. 7. Each of the ideas depicted was rejected as being impractical. Radiation coupling requires more surface area than is available in a reasonable length $(2 \mathrm{~m})$ of overlapping heat pipes. The one-on-one concept involves a complex annular heat pipe and a thermal bond that is difficult to achieve. The direct parallel coupling scheme requires that the core and converter heat pipes have either a rectangular or hexagonal cross section. The cross-coupling concept also requires rectangular 


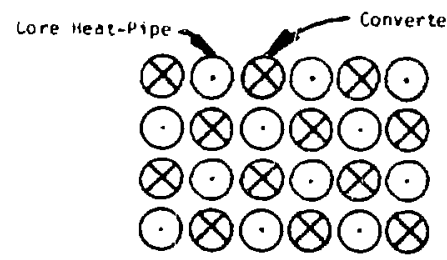

A. Radiant coupling

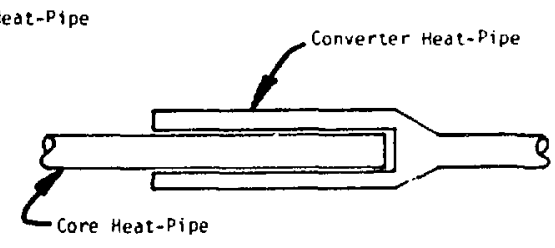

B. ONE-ON-ONE

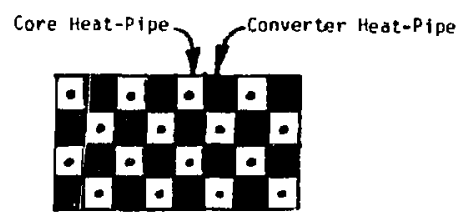

c. Parallel coupling

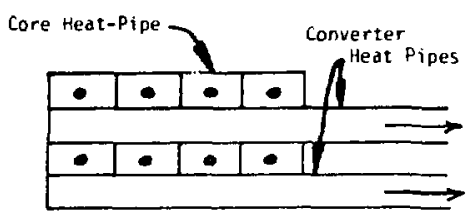

D. CROSS COUPLING

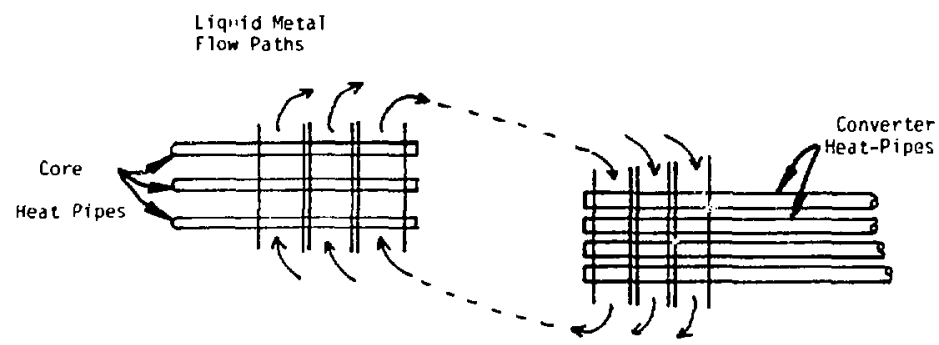

E. ReDUndant LIOUID-METAL LOOPS

Fig. 7. Several schemes for thermally coupling the core heat pipes to the thermionic converter heat pipes.

heat pipes and requires extra lengths of heat pipes (making it heavier) to turn the core and converter heat pipes so that they cross each other. The liquid-metal-loops heat exchanger is very heavy and requires pumps to circulate the liquid. The pumps require electrical power and tend to decrease the reliability of the system.

A heat exchanger design that appears practical and feasible is illustrated in Fig. 8. The figure shows a module of six core heat pipes that penetrate through two rectangular jackets, which are in reality two molybdenum coupling heat pipes. This assembly is mated with vertical bolts to a similar assembly containing a module of four converter heat pipes and two rectangular coupling heat pipes. The function of the coupling neat pipes is to transfer the heat from the core heat pipe module to the converter heat 
pipe module across the flat mating interface. Several coupling heat pipes are used to provide the desired amount of redundancy in case one should fail. Thin-wall sleeves internal to the coupling heat pipes allow the core or converter heat pipes to pass through. A braze is made after assembiy of each module to obtain a good thermal bond to the coupling heat pipes. The wick structure in the coupling heat pipes is so arranged as to provide liquid flow paths between the sleeves themselves and between the sleeves and the flat interface. For the core modules, the sleeves form the evaporator of the coupling heat pipes and the flat interface is the condenser. The roles are reversed for the converter modules.

The thermal bond between the core modules and the converter modules may be accomplished either with a deformable foil or by brazing when the reactor is brought up to power.

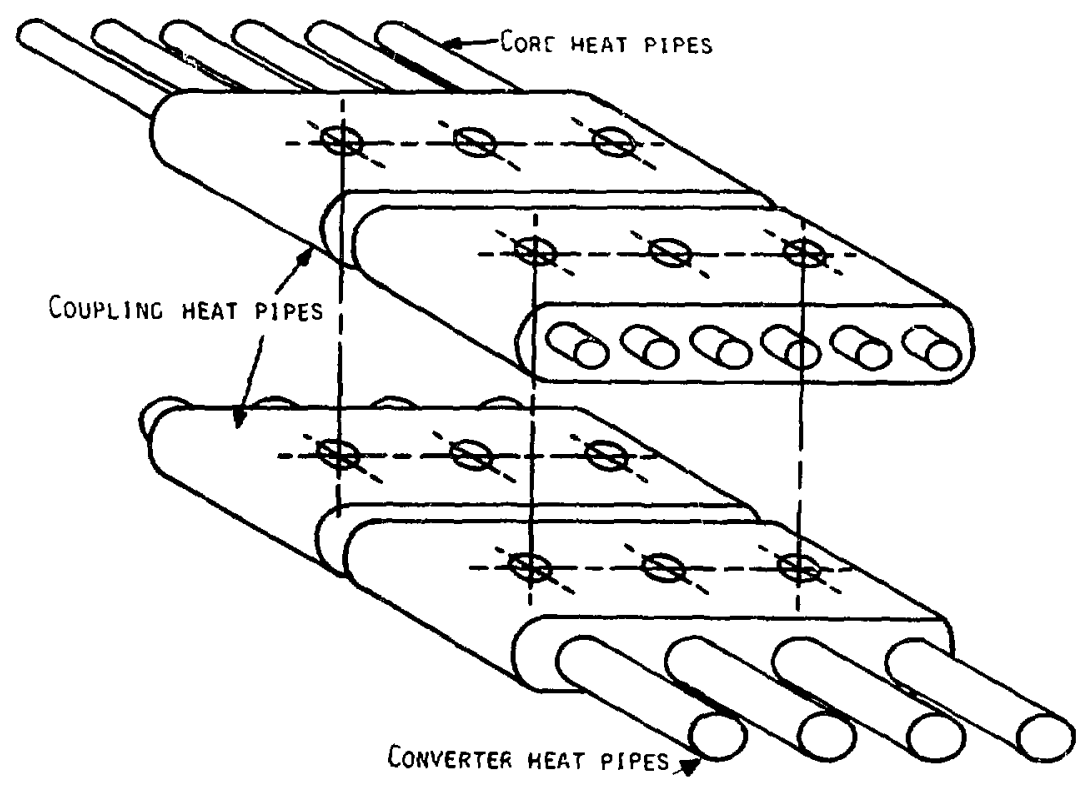

Fig. 8. Schematic design of coupling heat exchanger. 
An attractive feature of the concept is that the number and sizes of heat pipes in the core module, in the converter module and for the coupling heat pipes are all arbitrary and can be selected to satisfy the separate requirements of core and converter designs. There are other desirable features as well. The modular heat exchanger provides some cross coupling and some redundancy of heat flow paths. These features are important for smoothing out differences in the power carried by each heat pipe. Both core and converter heat pipes can be cylindrical. The mating surfaces are simple and do not require close alignment tolerances. The coupling can be performed before the core and converter modules are assembled together into a complete systeri. Or the coupling can be made after the core and the converter system have been assembled as separate units.

All the complexity of the heat exchanger design is concentrated in the coupling heat pipes. However, each coupling heat pipe can be tested separately prior to assembly into a module and each module can be tested for quality assurance prior to final assembly into the core or the converter system.

A preliminary design study of the heat exchanger was performed for a core containing 162 heat pipes arranged in 18 modules, each consisting of 9 core heat pipes and 6 coupling heat pipes. The thermionic converter unit contaired 90 heat pipes arranged in 18 modules of 5 converter heat pipes and 6 coupling heat pipes. The 18 pairs of modules were arranged in 6 rows of 3 pairs each. This choice of layout matches well with the current thermionic converter assembly. In principle, the 6 rows can be vertically stacked close together with bolts running through the entire height of the heat exchanger stack. This arrangement would require more careful alignment of the modules, sut it would simplify assembly and it would provide additional redundancy and better heat transfer because each core module internal to the stack would be in direct contact with two converter modules and vice versa. However, in the present analysis it was assumed for conservatism that there is only one good heat-transfer interface between pairs of core and converter modules.

The width of the hcat exchanger mentioned in the study refers to the overall width of one row. The length refers to the length of overlap of core and converter heat pipes. Both the length and the width of the heat exchanger were treated as variables in the parametric study. The height of the 
heat exchanger, closely stacked, was chosen to be $0.60 \mathrm{~m}$. This height corresponds to making the height of the coupling heat pipes approximately twice the diameter of the heat pipes running through them.

The estimates of weight for the heat exchanger were based on the following considerations: The wall thickness of the molybdenum coupling heat pipes was taken to be $0.5 \mathrm{~mm}$ including the wick. The weight of the internal sleeves was neglected. The combined weight of all the core and converter heat pipes was estimated to be $220 \mathrm{~kg} / \mathrm{m}$. The weight of Dolts and fittings for assembling the heat exchanger was assumed to be $26 \mathrm{~kg} / \mathrm{m}^{2}$ of projected area (length $x$ width of the heat exchanger).

The temperature drop attributable to the heat exchanger is the difference between the vapor temperature at the inlet to the condenser of the core heat pipes and the vapor temperature at the out let of the evaporator in the converter neat pipes. This temperature difference was assumed to contain only three components: the temperature drop across the wall of the core heat pipes, the temperature orop through the interface of the coupling heat pipes, and the temperature drop in the wall of the converter heat pipes. Temperature losses within the heat pipes were assumed to be negligible. Estimates for these temperature drops are listed in Table $V$. A plot of the temperature drop in the heat exchanger vs length is shown in Fig. 9 for several values of width. Similarly, a plot of heat exchanger mass versus length for several widths is shown in Fig. 10. Also included in

\section{TABLE $V$}

\section{TEMPERATURE DROPS IN 3-MW $\mathrm{t}$ HEAT EXCHANGER}

Location

Core heat-pipe wall, for $1 \mathrm{~m}$ length*

Converter heat pipe, for $1 \mathrm{~m}$ length*

Coupling heat-pipe interface, for $1 \mathrm{~m}^{2}$ of projected area
Temperature Drop (K)

12.5

0.39

22.4

0.43

18.8

0.50

\footnotetext{
*Temperature drop and heat flux are inversely proportional to length. \#Assumes contact area is one-third of projected area. Temperature drop and heat flux are inversely proportional to product of length $x$ width.
} 


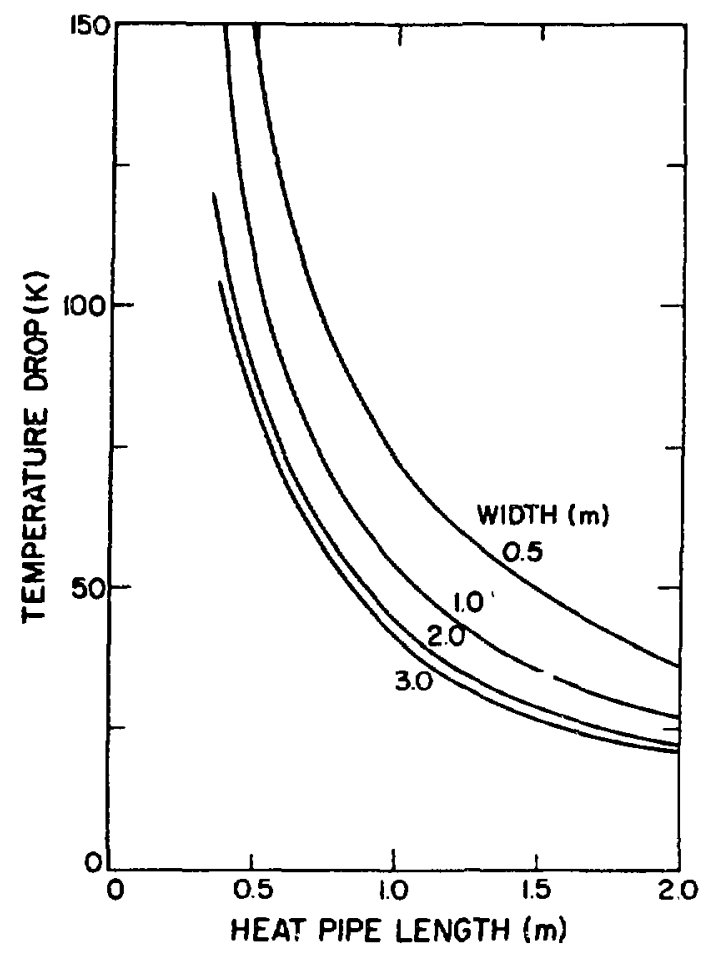

Fig. 9. Temperature drop in heat exchanger vs length of heat pipe overlap for several values of heat exchanger width.

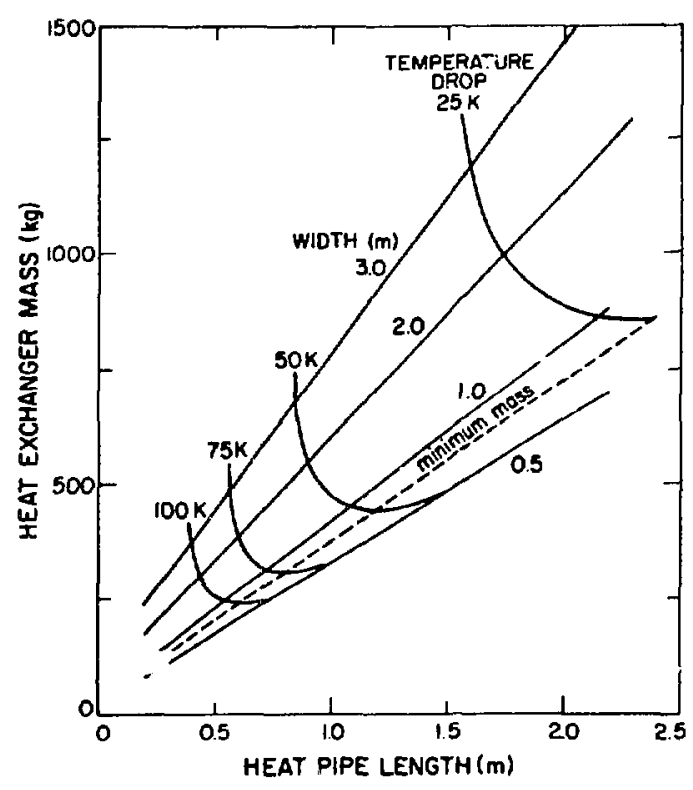

Fig. 10. Heat exchanger mass vs length for several values of heat exchange width. Also shown are curves of constant temperature drop in the heat exchanger. 
Fig. 10 shows curves of constant temperature drop. These latter curves show a minimum mass at a heat exchanger width of about $0.75 \mathrm{~m}$, indicated by the dashed line. Near the minimum, mass is rather insensitive to the heat exchanger width that can vary from 0.5 to $1.5 \mathrm{~m}$ and produce less than $10 \%$ variation in mass. The results shown in Figs. 9 and 10 are summarized in Fig. 11, which shows heat exchanger mass and length vs temperature drop for a constant width of $1 \mathrm{~m}$. This figure indicates, for example, that a $3 \mathrm{MW}_{\mathrm{T}}$ heat exchanger designed for a temperature drop of $50 \mathrm{~K}$ would weigh $450 \mathrm{~kg}$ and would fit in a volume $0.6 \mathrm{~m}$ high by $1 \mathrm{~m}$ wide by $1 \mathrm{~m}$ long.

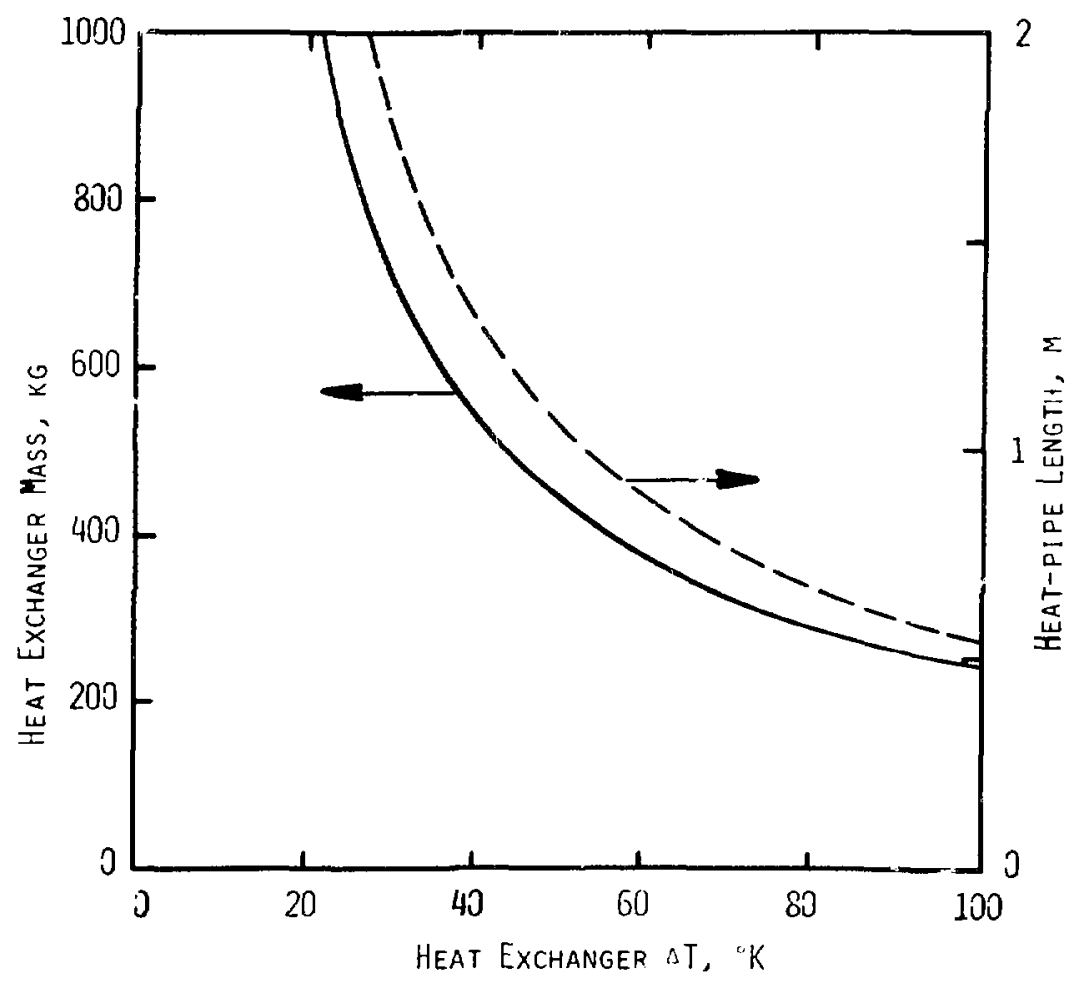

Fig. 11. Design purameters for 1-m-wide heat exchanger. 


\section{CONCLUSION}

The current status of the NEPS 3 MWT reactor shows the following:

- Detailed analysis of the effects of heat-pipe failures has indicated the desirability of raising the number of heat pipes in the core above 90 to, perhaps, 16z.

- Design parameters for a core containing 162 heat pipes were presented.

- As the result of a more realistic weight analysis, the core heat pipe weight has been reduced by nearly a factor of two.

- Special attention was given to the design of the core periphery and to the design of a new fuel element pctentially capable of containing in excess of $80 \mathrm{vol}_{2} \mathrm{UO}_{2}$ in t'e fuel region. This effort yielded solutions to the oifficulties caused by heat pipe failures on the core periphery.

- A conceptual design for a heat exchanger between the core and the thermionic converter assembly was described. This heat exchanger would provide design and fabrication decoupling for these two subassemblies but at an added mass perialty to the power plant on the order of $500 \mathrm{~kg}$. 